\title{
Polarization transfer in relativistic electron-nucleus bremsstrahlung
}

\author{
D. H. Jakubassa-Amundsen \\ Mathematics Institute, University of Munich, Theresienstrasse 39, \\ 80333 Munich, Germany
}

\begin{abstract}
The emission of circularly polarized photons during the scattering of fast spin-polarized electrons from heavy nuclei is studied within the Dirac-Sommerfeld-Maue approach. Predictions are made for the dependence of the polarization correlations $C_{32}$ and $C_{12}$ on collision energy, photon energy and nuclear charge. A comparison with pilot measurements of the transmission asymmetry, sensitive to $C_{32}$, for $3.5 \mathrm{MeV}$ e $+\mathrm{Pb}$ verifies that the polarization transfer increases with photon energy for small emission angles.
\end{abstract}

The precise knowledge of the polarization transfer from the beam particles to the photons emitted in a bremsstrahlung process, combined with an accurate measurement of the photon polarization via recently developed Compton polarimetry [1]-[4], provides a promising method to determine the degree of beam polarization and its change with time during an experiment [5]. Such knowledge is important for nuclear structure investigations. Bremsstrahlung may also be used as a source of radiation for a variety of applications, among those for the study of parity conservation in photofission experiments which requires a high degree of circular polarization of the photons [6]. Early theoretical predictions [7, 8], based on the Sommerfeld-Maue (SM) approximation introduced by Bethe and Maximon [9], indicated that the polarization transfer is particularly large at the short-wavelength limit (SWL). There, the electron transfers all its kinetic energy to the photon such that relativistic (spin) effects become highly important. These SM results for the polarization correlations were topped by accurate relativistic partial-wave calculation for a selection of collision systems [10]-[12], and only recently a systematic partial-wave study up to collision energies of $3 \mathrm{MeV}$ has become available [13]. At even higher energies the partial-wave theory (which then suffers from convergence problems) may be supplemented at the SWL by the Dirac-Sommerfeld-Maue (DSM) theory $[14,15]$ where the slow scattered electron is described by an exact Dirac state and the fast incoming electron by an SM function.

We consider the case where the target is a bare nucleus with charge number $Z$ and where the scattered electron is not observed. The doubly differential cross section $d \sigma$ for the emission of a photon with momentum $\boldsymbol{k}$, energy $\omega=k c$ and polarization $\boldsymbol{e}_{\lambda}$ into the solid angle $d \Omega_{k}$ is given (in atomic units, $\hbar=m=e=1)$ by $[16,17]$

$$
\begin{gathered}
d \sigma \equiv \frac{d^{2} \sigma}{d \omega d \Omega_{k}}=\frac{4 \pi^{2} \omega k_{f} E_{f}}{c^{3} v} \sum_{\sigma_{f}} \int d \Omega_{f}\left|\boldsymbol{e}_{\lambda}^{*} \boldsymbol{W}_{\text {rad }}\left(\sigma_{f}, \sigma_{i}\right)\right|^{2}, \\
\boldsymbol{e}_{\lambda}^{*} \boldsymbol{W}_{\text {rad }}\left(\sigma_{f}, \sigma_{i}\right)=\int d \boldsymbol{r} \psi_{f}^{\left(\sigma_{f}\right)+}(\boldsymbol{r})\left(\boldsymbol{\alpha} \boldsymbol{e}_{\lambda}^{*}\right) e^{-i \boldsymbol{k r}} \psi_{i}^{\left(\sigma_{i}\right)}(\boldsymbol{r}) .
\end{gathered}
$$

In (1), $\psi_{i}^{\left(\sigma_{i}\right)}$ and $\psi_{f}^{\left(\sigma_{f}\right)}$ describe, respectively, the initial and final electronic states with total energy $E_{i}$ and $E_{f}=E_{i}-\omega$, momentum $\boldsymbol{k}_{i}$ and $\boldsymbol{k}_{f}$ and spin projection $\sigma_{i}$ and $\sigma_{f}$. The vector $\boldsymbol{\alpha}$ comprises the Dirac 
matrices, $v$ is the collision velocity and $d \Omega_{f}$ the solid angle of the scattered electron. In the DSM theory, the partial-wave representation of the Dirac state $\psi_{f}^{\left(\sigma_{f}\right)}$ is employed. In order to avoid the inclusion of more partial waves, we resort to the SM theory when $\omega$ is sufficiently below the short-wavelength limit. In the SM theory $\psi_{i}^{\left(\sigma_{i}\right)}$ and $\psi_{f}^{\left(\sigma_{f}\right)}$ are both described by Sommerfeld-Maue functions, providing $\boldsymbol{W}_{\text {rad }}$ analytically.

Denoting the spin polarization vector of the incoming electron by $\boldsymbol{n}_{s}=\left(\zeta_{1}, \zeta_{2}, \zeta_{3}\right)$ and characterizing the photon polarization by $\xi_{k}, k=1,2,3$, Tseng and Pratt [11] parametrized the (doubly differential) cross section in the following way,

$$
\begin{gathered}
d \sigma=\frac{1}{2} d \sigma_{0}\left(1+C_{03} \xi_{3}+C_{11} \zeta_{1} \xi_{1}+C_{12} \zeta_{1} \xi_{2}+C_{20} \zeta_{2}+C_{23} \zeta_{2} \xi_{3}\right. \\
\left.+C_{31} \zeta_{3} \xi_{1}+C_{32} \zeta_{3} \xi_{2}\right),
\end{gathered}
$$

where $d \sigma_{0}$ is the cross section for unpolarized electrons (including a sum over the photon index $\lambda$ ). We choose a coordinate system with the $z$-axis $\left(\boldsymbol{e}_{z}\right)$ along the beam direction $\boldsymbol{k}_{i}$ and the $x$-axis $\left(\boldsymbol{e}_{x}\right)$ along $\boldsymbol{k}$ for the photon emission angle $\theta_{k}=90^{\circ} \quad\left(\boldsymbol{k}_{i}\right.$ and $\boldsymbol{k}$ define the reaction plane). The polarization $\boldsymbol{e}_{\lambda}$ can be written in terms of the basis vectors $\boldsymbol{e}_{\lambda_{1}}=(0,1,0)$ and $\boldsymbol{e}_{\lambda_{2}}=\left(-\cos \theta_{k}, 0, \sin \theta_{k}\right)$. Right $(+)$ and left $(-)$ circularly polarized photons are, respectively, represented by

$$
\boldsymbol{e}_{ \pm}=\frac{1}{\sqrt{2}}\left(\boldsymbol{e}_{\lambda_{2}} \pm i \boldsymbol{e}_{\lambda_{1}}\right)
$$

This definition of $\boldsymbol{e}_{ \pm}$corresponds to $\xi_{1}=\xi_{3}=0$ and $\xi_{2}= \pm 1$, such that for circularly polarized photons the parametrization (2) reduces to

$$
d \sigma=\frac{1}{2} d \sigma_{0}\left(1+C_{12} \zeta_{1} \xi_{2}+C_{20} \zeta_{2}+C_{32} \zeta_{3} \xi_{2}\right)
$$

Defining the spin polarization direction $\boldsymbol{n}_{s}$ with respect to the basis $\hat{\boldsymbol{\zeta}}_{1}=\boldsymbol{e}_{x}, \hat{\boldsymbol{\zeta}}_{2}=-\boldsymbol{e}_{y}, \hat{\boldsymbol{\zeta}}_{3}=-\boldsymbol{e}_{z}$ as done in our previous work [14], we can eliminate the polarization correlation $C_{20}$ from (4) by requiring that $\boldsymbol{n}_{s}$ lies in the reaction plane $\left(\zeta_{2}=0\right)$. Introducing the angle $\alpha_{s}$ between $\boldsymbol{n}_{s}$ and the $z$-axis, $\boldsymbol{n}_{s}$ can be written as $\boldsymbol{n}_{s}=\hat{\boldsymbol{\zeta}}_{1} \sin \alpha_{s}-\hat{\boldsymbol{\zeta}}_{3} \cos \alpha_{s}$ (such that in (4), $\zeta_{1}=\sin \alpha_{s}, \zeta_{3}=-\cos \alpha_{s}$ ).

The Stokes parameter $P_{3}$ for circularly polarized photons is defined by the asymmetry with respect to the emission of right- and left-circularly polarized photons,

$$
P_{3}\left(\alpha_{s}\right)=\frac{d \sigma\left(\boldsymbol{e}_{+}\right)-d \sigma\left(\boldsymbol{e}_{-}\right)}{d \sigma\left(\boldsymbol{e}_{+}\right)+d \sigma\left(\boldsymbol{e}_{-}\right)}=C_{12} \sin \alpha_{s}-C_{32} \cos \alpha_{s} .
$$

Thus the polarization correlations $C_{32}$ and $C_{12}$ can be calculated from

$$
C_{32}=-P_{3}(0) \equiv-P_{3 \|}, \quad C_{12}=P_{3}\left(90^{\circ}\right) \equiv P_{3 \perp},
$$

where the subscripts $\|$ and $\perp$ indicate that the electron spin is aligned with the beam axis, respectively, is perpendicular to it.

In Fig. 1 we show predictions for $C_{32}$ for bare $\mathrm{Au}$ and $\mathrm{Cu}$ targets in the collision energy range 5 $15 \mathrm{MeV}$ where the collision energy is defined by the kinetic energy $E_{i, k i n}=E_{i}-c^{2}$. The increase of $C_{32}$ with $E_{i, k i n}$ at the forward angles, already predicted by Haug [8] for low- $Z$ targets and supported by partial-wave results $[11,13]$ for $Z \leq 79$ in the energy range between $0.05-3 \mathrm{MeV}$, is seen to continue at the higher energies, and for $E_{i, k i n} \gtrsim 10 \mathrm{MeV}$, the polarization transfer at the SWL is complete at least up to $\theta_{k} \approx 120^{\circ}$. For a lower nuclear charge $C_{32}$ is slightly higher at the SWL, but it becomes independent of $Z$ for $\theta_{k} \rightarrow 0$. This $Z$-independence at $\theta_{k}=0$ holds also in the ultrarelativistic limit [7]. However, it is neither true at smaller collision energies (below, say, $1 \mathrm{MeV}[5,11]$ ) nor at the larger photon angles. When the photon energy gets smaller, $C_{32}$ strongly decreases in modulus and also its dependence on $E_{i, k i n}$ and $Z$ weakens considerably (Fig.1). 
In the experiments, performed with $3.5 \mathrm{MeV}$ electrons colliding with $\mathrm{Pb}$, the intensity difference for the emission of right- and left-circularly polarized photons is measured with the help of a magnetized absorber. The photons colliding with the polarized absorber electrons suffer Compton scattering, the cross section for which is polarization-dependent according to the Klein-Nishina formula [18]. The measured transmission asymmetry $A=\left(I_{+}-I_{-}\right) /\left(I_{+}+I_{-}\right)$where \pm corresponds, respectively, to right- and leftcircularly polarized photons, is thus not simply given by the product of $C_{32}$ with the polarization degree of the electron beam, but is in addition proportional to the polarization-dependent part of the Compton scattering cross section [3,5]. The upper theory curve in Fig.2 is a calculation of $A$ [3] using a beam polarization degree of 8 percent, the $C_{32}$ predictions from the ultrarelativistic Olsen formula [7] (neglecting the electron rest energy), and the scattering cross section based on the Klein-Nishina formula. In the lower theory curve, which is in better accord with the preliminary data points, the Olsen predictions for $C_{32}$ are replaced by calculations of $C_{32}$ within the DSM model (at $\omega=3.5 \mathrm{MeV}$ ), respectively with the SM theory (for the lower $\omega$ ).

When the electrons are spin-polarized perpendicular to the beam axis, such that the polarization transfer is given in terms of the polarization correlation $C_{12}$, the angular dependence changes dramatically. As seen from Fig.3, $C_{12}$ does not only vanish at $\theta_{k}=0$ and $180^{\circ}$ (like all other polarization correlations except $C_{32}$ [11]), but at the SWL it exhibits a double-well structure with a strong minimum at the backmost angles, which is the deeper, the higher the collision energy. We expect, however, that at energies exceeding $15 \mathrm{MeV}$ this minimum will shrink again similar to the case of the spin asymmetry correlation $C_{20}$ [14] when going beyond $10 \mathrm{MeV}$. As concerns the maximum at small angles, ultrarelativistic estimates provide the bound $C_{12} \lesssim 0.1$ [20] in agreement with our results. Also the $Z$-dependence of $C_{12}$ is much more pronounced than for $C_{32}$.

The accuracy of the present calculations for the polarization correlation $C_{32}$ related to longitudinally spin-polarized electrons is quite satisfactory. This may be inferred from Fig. 4 where for a gold target the DSM results at $E_{i, k i n}=\omega=3 \mathrm{MeV}$ are compared with the screened-atom partial-wave results of Yerokhin and Surzhykov [13]. We recall that for the polarization correlations screening effects are unimportant [11], being at most 10 percent for impact energies between $0.1-1 \mathrm{MeV}$, and decreasing with $E_{i, k i n}$ beyond $1 \mathrm{MeV}$ [13]. Even in the case of $E_{i, k i n}=1 \mathrm{MeV}$ and $\omega=0.9 \mathrm{MeV}$ (and more so for decreasing $\omega)$ the differences between the SM approximation and the partial-wave results are small. This result is somewhat unexpected, taken into consideration that the Sommerfeld-Maue functions, particularly for electrons close to the nucleus, are inappropriate at low energies [19]. In fact, for the Stokes parameter $P_{2}$, related to linearly polarized photons, a systematic comparison with partial-wave results has shown that the SM functions become only reliable at sufficiently high electron energies $E$ such that $Z c / E \lesssim 0.02$ [15]. For $C_{12}$ the present calculations indeed are in severe disagreement with partial-wave results in a situation comparable to Fig.3 (3 MeV e $+\mathrm{Au}, \omega=1.5 \mathrm{MeV}[21])$. We therefore expect that, for a gold target, $E_{i, k i n} \gtrsim 15 \mathrm{MeV}$ is needed for an adequate representation of $C_{12}$ within the DSM model.

In conclusion, we have applied the Dirac-Sommerfeld-Maue theory (and for less energetic photons the SM theory) for predicting the angular dependence of the polarization correlations related to circularly polarized photons and electrons spin-polarized in the reaction plane. It was shown that for $C_{32}$, describing the polarization transfer from longitudinally spin-polarized electrons, the DSM respective SM theory performs well even for heavy targets at energies down to $1 \mathrm{MeV}$. Also the measured dependence of the photon transmission asymmetry on the photon energy for $3.5 \mathrm{MeV}$ longitudinally spin-polarized electrons colliding with lead is well reproduced. Experiments with fast, transversely spin-polarized electrons, sensitive to $C_{12}$, are highly welcome to test the theoretical predictions.

\section{Acknowledgments}

It is a pleasure to thank R.Barday for stimulating this project and for valuable discussions. I am also very grateful to F.Nillius and K.Aulenbacher for the communication of experimental results and calculations prior to publication, and to V.A. Yerokhin for performing additional partial-wave calculations. 


\section{References}

[1] S.Tashenov et al, Phys. Rev. Lett. 97 (2006) 223202.

[2] S.Tashenov, T.Bäck, R.Barday, B.Cederwall, J.Enders, A.Khaplanov, Yu. Poltoratska, K.U.Schässburger, A.Surzhykov, submitted for publication.

[3] F.Nillius, K.Aulenbacher, PESP Proceedings (2010).

[4] R.Märtin et al, Contributed paper to CAARI, Denton, Texas (2010).

[5] R.Barday et al, PESP Proceedings (2010).

[6] S.Tashenov, private communication.

[7] H.Olsen, L.C.Maximon, Phys. Rev. 114 (1959) 887.

[8] E.Haug, Phys. Rev. 188 (1969) 63.

[9] H.A.Bethe, L.C.Maximon, Phys. Rev. 93 (1954) 768.

[10] H.Brysk, C.D.Zerby, S.K.Penny, Phys. Rev. 180 (1969) 104.

[11] H.K.Tseng, R.H.Pratt, Phys. Rev. A 7 (1973) 1502.

[12] I.J.Feng, I.B.Goldberg, Y.S.Kim, R.H.Pratt, Phys. Rev. A 28 (1983) 609.

[13] V.A.Yerokhin, A.Surzhykov, Phys. Rev. A 82 (2010) 062702.

[14] D.H.Jakubassa-Amundsen, Phys. Rev. A 82 (2010) 042714.

[15] D.H.Jakubassa-Amundsen, A.Surzhykov, Eur. Phys. J. D (2011) in print.

[16] W.Heitler, Quantum Theory of Radiation, Clarendon Press, Oxford, 1954, $3^{\text {rd }}$ edition, $§ 25$.

[17] H.K.Tseng, R.H.Pratt, Phys. Rev. A 3 (1971) 100.

[18] O.Klein, Y.Nishina, Z. Phys. 52 (1929) 853; Y.Nishina, Z. Phys. 52 (1929) 869.

[19] J.K.Fink, R.H.Pratt, Phys. Rev. A 7 (1973) 392.

[20] H.Olsen, L.C.Maximon, Phys. Rev. 110 (1958) 589.

[21] V.A.Yerokhin, private communication.

\section{Figure captions}

Fig.1

Polarization correlation $C_{32}$ for longitudinally spin-polarized electrons as a function of photon angle $\theta_{k}$. a) $\mathrm{Au}^{79+}$ : upper curves, SWL (DSM results) for beam energy $E_{i, k i n}=5 \mathrm{MeV}(\longrightarrow$ ) $), 10 \mathrm{MeV}$ $(-\cdot-\cdot)$ and $15 \mathrm{MeV}(----)$. Lower curves, $\omega / E_{i, k i n}=\frac{1}{5}$ (SM results) for $E_{i, k i n}=5 \mathrm{MeV}(-)$ and $10 \mathrm{MeV}(-\cdot-\cdot-)$.

b) $\mathrm{Au}^{79+}(-)$ and $\mathrm{Cu}^{29+}(-\cdot-\cdot-)$ for $5 \mathrm{MeV}$ : uppermost curves, SWL (DSM results); middle curves, $\omega=3 \mathrm{MeV}\left(\omega / E_{i, k i n}=\frac{3}{5}\right)$, lowermost curves, $\omega=1 \mathrm{MeV}\left(\omega / E_{i, k i n}=\frac{1}{5}\right)$ (all SM results).

Fig.2

Transmission asymmetry $A$ (in percent) for $3.5 \mathrm{MeV}$ longitudinally spin-polarized electrons colliding with a lead target as a function of photon energy $\omega$ for $\theta_{k}=0$. The experimental data $(\bullet)$ are from Nillius and 
Aulenbacher [3], calculations from Nillius and Aulenbacher using Olsen's formula for $C_{32}(----)$, and scaled down by the ratio $C_{32}$ (present) $/ C_{32}$ (Olsen)

Fig.3

Polarization correlation $C_{12}$ for transversely spin-polarized electrons at the SWL as a function of photon angle $\theta_{k} \cdot \mathrm{Au}^{79+}: 5 \mathrm{MeV}(-), 10 \mathrm{MeV}(-\cdot-\cdot-), 15 \mathrm{MeV}(----) \cdot \mathrm{Cu}^{29+}: 5 \mathrm{MeV}(\cdots \cdots \cdots)$ (DSM results).

Fig.4

Polarization correlation $C_{32}$ for a gold target as a function of photon angle $\theta_{k}$. Shown are (screenedtarget) partial-wave results (- from [13] for $3 \mathrm{MeV}$ electrons at the SWL $(\omega=3 \mathrm{MeV}$, upper curve), for $1 \mathrm{MeV}$ electrons and photons of energy $\omega=0.9 \mathrm{MeV}$ (middle curve) and $\omega=0.5 \mathrm{MeV}$ (lower curve), as well as results (for $\mathrm{Au}^{79+}$ ) within the DSM model $\left(-\cdot-\cdot-\right.$, SWL at $E_{i, k i n}=3 \mathrm{MeV}$ ) and the $\mathrm{SM}$ approach $\left(E_{i, \text { kin }}=1 \mathrm{MeV}\right.$ and,$\left.---- \omega=0.9 \mathrm{MeV} ; \cdots \cdots, \omega=0.5 \mathrm{MeV}\right)$. 